Lenke H, Achtnich C, Daun G, Knackmuss HJ (2000): Bioremediation of TNT-contaminated soil. In: Wise DL, Trantolo DJ, Cichon EJ, Inyang HI, Stottmeister U, eds: Bioremediation of contaminated soils. Marcel Dekker, New York, Basel, 561-578

LfU (1991) Landesanstalt für Umweltschutz Baden-Württemberg: Handbuch Mikrobiologische Bodenreinigung. Materialien zur Altlastenbearbeitung. 7, $233 \mathrm{~S}$

Lorenzen T (2000): Nematoden als Bioindikatoren einer in-situ Phytoremediation TNT-belasteter Böden (Werk Tanne, Clausthal-Zellerfeld). Diplomarbeit Universität Bremen, FB 2

Marwood TM, Knoke K, Yau K, Lee H, Trevors JT, SuchorskiTremblay A, Flemming CA, Hodge V, Liu DL, Seech AG (1998): Comparison of toxicity detected by five bioassays during bioremediation of diesel fuel-spiked soils. Environ. Toxicol. Water Qual. 13, 117-126

Meharg AA, Denis GR, Cairney JWG (1997): Biotransformation of 2,4,6-trinitrotoluene (TNT) by ectomycorrhizal basidiomycetes. Chemosphere 35, 513-521

Melchior S (2000): Materialwahl, Schichtaufbau und Dimensionierung der Rekultivierungsschicht. Hamburger Bodenkdl. Arbeiten 47, 191-216

Moormann H (2001): Einfluß der Rhizodeposition von Helophyten auf den mikrobiellen Schadstoffabbau. Dissertation Univ. Bremen, FB 2, Okt. 2001

Oades JM (1993): The role of biology in the formation, stabilization and degradation of soil structure. Geoderma 56,377-400

Parmelee RW (1995): Soil fauna: linking different levels of ecologi-

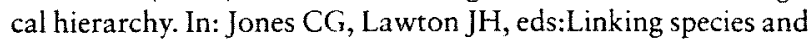
ecosystems. Chapman \& Hall, N.Y., London, 107-116

Parmelee RW, Wentsel RS, Phillips CT, Simini W, Checkai RT (1993): Soil microcosm for testing the effects of chemical pollutants on soil fauna communities and trophic structure. Environ. Toxicol. Chem. 12, 1477-1486

Peterson MM, Horst GL, Shea PJ, Comfort SD (1998): Germination and seedling developmenet of switchgrass and smooth brome grass exposed to 2,4,6-trinitrotoluene. Environ. Poll. 99, 53-59

Scheibner K, Hofrichter M, Herre A, Michels J, Fritsche W (1997): Screening for fungi intensively mineralizing 2,4,6-trinitrotoluene. Appl. Microbiol. Biotechnol. 47, 452-457

Scheidemann P, Klunk A, Sens C, Werner D (1998): Species Dependent Uptake and Tolerance of Nitroaromatic Compounds by Higher Plants. J. Plant Physiol. 152, 242-247

Schnoor JL, Licht SC, McCutcheon SC, Wolfe NL, Carreira LH (1995): Phytoremediation of organic and nutrient contaminants. Environ. Sci. Technol. 29, 318-323

Schönmuth B, Pestemer W (2000): Aufnahme und Verbleib von $\mathrm{C}^{14}$-Trinitrotoluol bei Weiden und Fichten. Tagungsband 2. Symposium Natural Attenuation, 7.-8. 12. 2000, DECHEMA
Schwab AP (1998): Phytoremediation of soils contaminated with PAHs and other petroleum compounds. Presented at: Beneficial effects of vegetation in contaminated soils, Kansas State University, Manhattan, KS, January 7-9, 1998

Shimp JF, Tracy JC, Davis LC, Lee E, Huang W, Erickson LE, Schnoor JL (1993): Beneficial effects of plants in the remediation of soil and groundwater contaminated with organic materials. Crit. Rev. Environ. Sci. Technol. 23, 41-77

Simini M, Wentsel RS, Checkai RT, Phillips CT, Chester NA, Major MA, Amos JC (1995): Evaluation of soil toxicity at Joliet Army Ammunition Plant. Environ. Toxicol. Chem. 14, 623-630

Sunahara GI, Dodard S, Sarrazin M, Paquet L, Ampleman G, Thiboutot S, Hawari J, Renoux AY (1998): Development of a soil extraction procedure for ecotoxicity characterization of energeric compounds. Ecotoxicol. Environ. Saf. 39, 185-194

Thomas H, Gerth, A, Eulering, B, Böhler A (2001): Neue Erkenntnisse zur biologischen In-situ-Sanierung TNT-kontaminierter Böden. TerraTech 2/2001, 52-54

Thompson PL, Ramer LA, Schnoor JL (1998): Uptake and Transformation of TNT by hybrid Poplar Trees. Environ. Sci. Technol. $32,975-980$

Trapp S (2000): Aspekte der Phytoremediation organischer Schadstoffe. UWSF - Z Umweltchem Ökotox 12, 246-255

Van Gestel CAM, Van der Waarde JJ, Derksen JGM, Van der Hoek EE, Veul MFXW, Bouwens S, Rusch B, Kronenburg R, Stokman GNM (2001): The use of acute and chronic bioassays to determine the ecological risk and bioremediation efficiency of oilpolluted soils. Environ. Toxicol. Chem. 20, 1438-1449

Vitousek PM, Reiners WA (1975): Ecosystem succession and nutrient retention: a hypothesis. Bioscience 25: 376-381

Warrelmann J, Koehler H, Frische T, Dobner I, Walter U, Heyser W (2000a): Erprobung und Erfolgskontrolle eines Phytoremediationsverfahrens zur Sanierung Sprengstoff-kontaminierter Böden: I. Konzeption und Einrichtung eines Freilandexperimentes. UWSF - Z Umweltchem Ökotox 12, 351-357

Warrelmann J, Behrend P, Koehler H (2000b): Monitoring TNTcontent of soils during phytoremediation. Proc. ConSoil 2000: 1176-1177

Wolff HJ (1998): Prüf- und Eingreifwerte für die Beurteilung einer Boden-/Grundwasserkontamination mit sprengstofftypischen Schadstoffen. Weiterbildungsseminar der Johannes GutenbergUniversität Mainz am 4.-6. März 1998: Rüstungsaltlasten und militärchemische Stoffe, $15 \mathrm{~S}$

Eingegangen: 26. 10. 2001

Akzeptiert: 05. 11.2001

OnlineFirst: 07.11. 2001

In UWSF 1/2002 (Januar-Ausgabe) erscheint

\title{
Phytovolatilisation organischer Chemikalien
}

\section{Oliver Baeder-Bederski-Anteda}

\section{DOI: http://dx.doi.org/10.1065/uwsf2001.11.073}

Zusammenfassung. Bei der Sanierung kontaminierter Böden und Wässer mit Hilfe von Pflanzen sind eine Vielzahl teilweise noch nicht quantifizierter Eliminationspfade wirksam. Unter 'Phytovolatilisation' versteht man die Emission meist organischer Stoffe aus den oberirdischen Teilen der Pflanze in die Atmosphäre. Damit werden alle Stoffe einbezogen, die über die Wurzel aufgenommen, teilweise transformiert und in den Spross transportiert werden. Die Phytovolatilisation ist vor allem bei der Elimination flüchtiger Stoffe oberflächennaher Kontaminationen von praktischem Interesse. In Gasaustausch-Experimenten wurde die Dynamik dieser Emissionen untersucht und an Hand eines Modells geprüft, welche Stoffe auf Grund ihrer physikalischen Eigenschaften durch Pflanzen emittiert werden können. 